\title{
Solving Maxwell equations in 3D prismatic domains
}

\author{
Patrick Ciarlet Jr. ${ }^{\text {a }}$, Emmanuelle Garcia ${ }^{\text {a }}$, Jun Zou ${ }^{\text {b }}$ \\ a ENSTA-CNRS-INRIA UMR 2706 POEMS, 32, boulevard Victor, 75739 Paris cedex 15, France \\ ${ }^{\mathrm{b}}$ Department of Mathematics, The Chinese University of Hong Kong, Shatin, N.T., Hong Kong \\ Received 2 September 2004; accepted 28 September 2004
}

Presented by Roland Glowinski

\begin{abstract}
In this Note, we introduce the Fourier Singular Complement Method, for solving Maxwell equations in a 3D prismatic domain. The numerical implementation of this method provides a continuous approximation of the electromagnetic field. It can be applied to the computation of propagating and evanescent modes in prismatic stub filters, thus generalizing 2D approaches. To cite this article: P. Ciarlet, Jr., et al., C. R. Acad. Sci. Paris, Ser. I 339 (2004).

(C) 2004 Académie des sciences. Published by Elsevier SAS. All rights reserved.
\end{abstract}

\section{Résumé}

Résolution des équations de Maxwell dans des domaines prismatiques tridimensionnels. Dans cette Note, nous introduisons la Méthode du Complément Singulier avec Fourier, pour résoudre les équations de Maxwell dans des domaines prismatiques tridimensionnels. La mise en œuvre numérique de cette méthode permet de calculer une approximation continue du champ électromagnétique. Elle peut être appliquée à la détermination des modes propagatifs ou bloquants dans un filtre à stubs prismatique, ce qui constitue une généralisation des méthodes applicables en domaine bidimensionnel. Pour citer cet article : P. Ciarlet, Jr., et al., C. R. Acad. Sci. Paris, Ser. I 339 (2004).

(C) 2004 Académie des sciences. Published by Elsevier SAS. All rights reserved.

\section{Version française abrégée}

Les nombres entre parenthèses renvoient à la version anglaise. L'opérateur rotationnel est noté curl.

Nous nous intéressons à la résolution des équations de Maxwell dans un domaine $\Omega$ prismatique, i.e. $\Omega=$ $\omega \times] 0, L[$ (voir la Fig. 1), à l'aide d'une généralisation de la méthode du Complément Singulier, bien connue en domaine $2 \mathrm{D}[2,1,10,8,11]$. Elle fournit une discrétisation continue du champ électromagnétique, avec prise en compte explicite des conditions aux limites. On souhaite simuler la propagation d'ondes électromagnétiques autour

\footnotetext{
E-mail addresses: Patrick.Ciarlet@ensta.fr (P. Ciarlet, Jr.), garciaemm@yahoo.fr (E. Garcia), zou@math.cuhk.edu.hk (J. Zou).
} 
d'un conducteur parfait. On se ramène à un domaine borné, en introduisant une frontière artificielle, notée $\Gamma_{A}$, sur laquelle on impose une condition aux limites de type Silver-Müller. Il est important de noter que le champ est régulier au voisinage de cette frontière artificielle [6]. La frontière du domaine de calcul $\Omega$ est donc décomposée en $\partial \Omega=\overline{\Gamma_{C}} \cup \overline{\Gamma_{A}}$; on appelle $\mathbf{n}$ la normale unitaire extérieure à $\partial \Omega$. Soit $(\mathcal{E}, \mathcal{H})$ le champ électromagnétique, fonction de $\mathbf{x}$ et $t$. Les équations de Maxwell dans le vide sont relatées en (1) et (2). Les densités de charge $\rho$ et de courant $\mathcal{J}$ vérifient l'équation de conservation de la charge $\partial_{t} \rho+\operatorname{div} \mathcal{J}=0$, et la connaissance de $\mathbf{e}^{\star}$ permet de modéliser les ondes planes incidentes $\left(\mathbf{e}^{\star} \neq 0\right)$, ou la frontière absorbante $\left(\mathbf{e}^{\star}=0\right)$. A la suite de $[3,4]$, on peut reformuler de façon équivalente (1), (2), en remplaçant les deux premières équations de (1) par (3), (4), ce qui permet d'obtenir deux systèmes «découplés » du second ordre en temps, en $\mathcal{E}$ et en $\mathcal{H}$. Dans la suite, on considère uniquement le système en $\mathcal{E}$, sachant que celui en $\mathcal{H}$ peut être résolu similairement. On peut prouver (cf. [4]) que, sous des hypothèses de régularités des données ad hoc, le champ $\mathcal{E}$ est l'unique solution de la Formulation Variationnelle (6), de régularité en temps (7). Si on suppose que la densité de charge appartient à $\mathbf{L}^{2}(\Omega)$, on peut «stabiliser» cette $\mathrm{FV}$ par ajout d' un terme en $(\operatorname{div} \cdot \operatorname{div} \cdot)_{0}$ et dualisation de la relation de Coulomb [3] : on aboutit à une FV Augmentée, Mixte (9), (10). Elle est posée dans l'espace produit $\mathcal{X}_{E} \times L^{2}(\Omega)$ (voir (8)); le multiplicateur de Lagrange est introduit pour imposer l'équation de Coulomb, dans le cas où l'équation de conservation de la charge ne serait pas vérifiée numériquement (par exemple, calcul du déplacement de particules chargées soumises au champ auto-généré).

Comme on l'a remarqué précédemment, le champ est régulier au voisinage de la frontière artficielle $\Gamma_{A}$. Ceci signifie que, modulo un relèvement $\mathbf{H}^{1}(\Omega)$, on peut toujours supposer que le champ électrique appartient à l'espace $\mathcal{X}_{E}^{0}$ (voir (8)). Or, à l'aide de [5,7], on peut facilement prouver la

Proposition 0.1. Soit $\mathcal{V} \in \mathcal{X}_{E}^{0}: \mathcal{V}_{z}$ appartient à $H^{1}(\Omega)$, et $\partial_{z} \mathcal{V}_{x}$ et $\partial_{z} \mathcal{V}_{y}$ appartiennent toutes deux $\grave{a} L^{2}(\Omega)$.

En d'autres termes, dans un domaine prismatique $\Omega=\omega \times] 0, L\left[\right.$, seuls $\mathcal{E}_{x}$ et $\mathcal{E}_{y}$ peuvent avoir un comportement singulier et, qui plus est, uniquement selon les variables $x$ et $y$. Si on tient compte des conditions aux limites sur $\mathcal{E}$, on remarque que l'on peut décomposer ses trois composantes selon (11) dans $\mathbf{L}^{2}(\Omega)$, ce qui induit les expressions de son rotationnel (12), (13) et de sa divergence (14) (resp. dans $\mathbf{L}^{2}(\Omega)$ et $L^{2}(\Omega)$ ). On choisit enfin la décomposition (15) pour $p$. On introduit alors les opérateurs $2 \mathrm{D} \operatorname{curl} \mathbf{v}=\partial_{x} v_{y}-\partial_{y} v_{x}$ et div $\mathbf{v}=\partial_{x} v_{x}+\partial_{y} v_{y}$, ainsi que $v$ la normale unitaire extérieure à $\partial \omega$, et $\tau$ le vecteur unitaire tel que $(\tau, v)$ est directe. On décompose la frontière $\partial \omega$ en $\partial \omega=\overline{\gamma_{C}} \cup \overline{\gamma_{A}}$, et on définit les espaces de Sobolev $\mathbf{X}_{E}$ et $X_{E}(16)$, «traces »sur $\omega$ de $\mathcal{X}_{E}$. A l'aide de séries de Fourier adéquates pour $\mathcal{J}, \rho$ et $\overrightarrow{\mathbf{e}}^{\star}$, on vérifie que (9), (10) est équivalente à (18), (19).

Ceci va nous permettre de définir la Méthode du Complément Singulier avec Fourier, pour résoudre numériquement le problème posé. D'après la Proposition 0.1, il est loisible d'approcher $\mathcal{E}_{z}^{k}$ à l'aide de l'EF de Lagrange continu $P_{2}$ (par exemple). Pour ce qui concerne les deux premières composantes $\mathcal{E}_{x, y}^{k}$, nous choisissons parmi les MCS $[2,1,10,8,11]$, l'EF de Lagrange continu $P_{2}$ (composante par composante), avec un Complément Singulier. Enfin, $p$ est approché à l'aide de l'EF de Lagrange continu $P_{1}$. Il en résulte un EF de type Taylor-Hood, avec Complément Singulier. Outre la coercivité de la forme bilinéaire sur $\left(\mathbf{X}_{E} \times X_{E}\right)^{2}$ (pour tout $k \geqslant 0$ ), on prouve aisément la

Proposition 0.2. La FVAM 2D discrète (18), (19) vérifie une condition discrète inf-sup uniforme.

NB. Cette condition est uniforme vis-à-vis du pas du maillage et de l'indice de Fourier.

Pour conclure, nous définissons l'approximation par la Méthode du Complément Singulier avec Fourier de $(\mathcal{E}, p)$. Soit $h$ le pas du maillage de la triangulation de $\omega$, et $N$ le nombre de modes Fourier effectivement calculés. Suivant [7] et ce qui précède, nous approchons $\mathcal{E}$ et $p$ par (20). 


\section{Introduction}

The Singular Complement Method (SCM) was originally developed to solve Maxwell equations in bounded nonconvex domains, with a piecewise smooth boundary. Indeed, when the domain is either convex or with a smooth boundary, all components of the electromagnetic field belong to $H^{1}(\Omega)$. This feature guarantees the convergence of Finite Element techniques, based on the continuous $P_{1}$ or $P_{2}$ Lagrange Finite Element approximation [3]. But when there are geometrical singularities, such as reentrant corners in $2 \mathrm{D}$, or reentrant edges and/or nonconvex vertices in 3D, the components of the EM field do not belong in general to $H^{1}(\Omega)$. What is more, the subspace of all $\mathbf{H}^{1}(\Omega)$-smooth fields is closed in the space of admissible EM fields. Since the continuous Lagrange FE approximation always belongs to the regular subspace, this prevents convergence (see [2]). To address this difficulty, one possibility is to enrich the space of test-fields by singular fields: we call this approach the Singular Complement Method. We refer to $[2,1,10,8,11]$ for instances of the 2D-SCM. Interestingly, this method works for both the static and time-dependent Maxwell equations [2,1,9].

This Note explains how one can apply a similar technique to solve Maxwell equations in a 3D prismatic domain.

\section{The model problem and its variational formulation}

The aim is to compute the EM field, in the neighborhood of a perfect conducting body $\mathcal{O}$, which is locally of prismatic shape. In order to reach this goal, we define a computational domain $\Omega$, by taking the complementary of $\mathcal{O}$ in a cube (for instance): its boundary $\partial \Omega$ is made up of two parts: $\Gamma_{C}$ and $\Gamma_{A}$, with $\overline{\Gamma_{C}}=\partial \Omega \cap \partial \mathcal{O}$ the perfect conductor boundary (the interface with the body $\mathcal{O}$ ), and $\Gamma_{A}$ an artificial boundary, which is introduced in order to bound the domain. It is split into $\Gamma_{A}^{i}$ and $\Gamma_{A}^{a}$. On $\Gamma_{A}^{i}$, we model incoming plane waves, whereas we impose on $\Gamma_{A}^{a}$ an absorbing boundary condition, both with the help of the Silver-Müller condition. The domain $\Omega$ is equal to $\Omega=\omega \times] 0, L[$, where $\omega$ is a $2 \mathrm{D}$ polygon (see the Fig. 1). So, the geometrical singularities are the reentrant edges of $\Gamma_{C}$, i.e. those edges with an interior dihedral angle larger than $\pi$. It is crucial to note that the EM field is smooth in a neighborhood of $\Gamma_{A}$ [6], so its singular behaviour occurs only at the reentrant edges of $\Gamma_{C}$.

Let $\mathcal{E}$ and $\mathcal{H}$ be the electric and magnetic fields, which depend on the space variable $\mathbf{x}$ and on the time variable $t$. Further, let $c, \varepsilon_{0}$ and $\mu_{0}$ be respectively the light velocity, the dielectric permittivity and the magnetic permeability $\left(\varepsilon_{0} \mu_{0} c^{2}=1\right)$. Then, Maxwell equations in vacuum, supplemented with boundary and initial conditions, can be expressed:

$$
\begin{aligned}
& \varepsilon_{0} \frac{\partial \mathcal{E}}{\partial t}-\operatorname{curl} \mathcal{H}=-\mathcal{J}, \quad \mu_{0} \frac{\partial \mathcal{H}}{\partial t}+\operatorname{curl} \mathcal{E}=0, \quad \operatorname{div}\left(\varepsilon_{0} \mathcal{E}\right)=\rho, \quad \operatorname{div}\left(\mu_{0} \mathcal{H}\right)=0 \quad \text { in } \Omega, \\
& \mathcal{E} \times \mathbf{n}=0 \quad \text { on } \Gamma_{C}, \quad\left(\mathcal{E}-\sqrt{\frac{\mu_{0}}{\varepsilon_{0}}} \mathcal{H} \times \mathbf{n}\right) \times \mathbf{n}=\mathbf{e}^{\star} \times \mathbf{n} \quad \text { on } \Gamma_{A}, \quad \mathcal{E}(0)=\mathcal{E}_{0}, \quad \mathcal{H}(0)=\mathcal{H}_{0} .
\end{aligned}
$$

The data $\rho$ and $\mathcal{J}$ are the charge and current densities (they satisfy the charge conservation equation $\partial_{t} \rho+\operatorname{div} \mathcal{J}=$ $0)$, $\mathbf{e}^{\star}$ models the incoming plane waves $\left(\mathbf{e}^{\star} \neq 0\right)$ or the absorbing b. c. $\left(\mathbf{e}^{\star}=0\right)$, and $\left(\mathcal{E}_{0}, \mathcal{H}_{0}\right)$ is the initial value

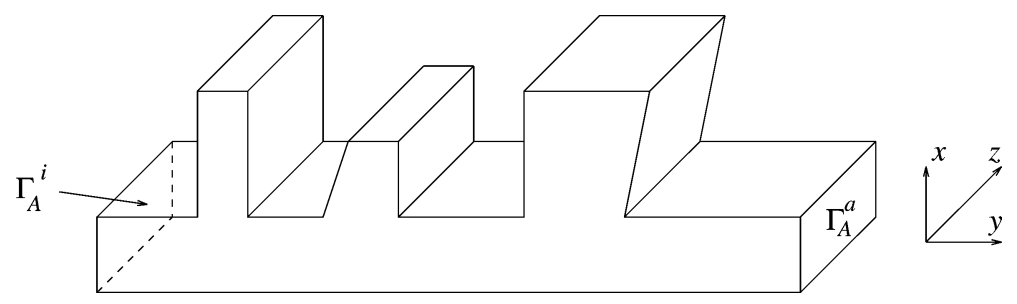

Fig. 1. Sample prismatic domain. 
of the EM field. Following for instance [3,4], one can equivalently reformulate (1), (2) as two second order in time systems of equations, the first one in $\mathcal{E}$, the second one in $\mathcal{H}$. One replaces the first two equations of (1) by

$$
\begin{aligned}
& \frac{\partial^{2} \mathcal{E}}{\partial t^{2}}+c^{2} \operatorname{curl} \operatorname{curl} \mathcal{E}=-\frac{1}{\varepsilon_{0}} \frac{\partial \mathcal{J}}{\partial t}, \quad \frac{\partial^{2} \mathcal{H}}{\partial t^{2}}+c^{2} \operatorname{curl}(\operatorname{curl} \mathcal{H}-\mathcal{J})=0 \quad \text { in } \Omega, \\
& \frac{1}{\varepsilon_{0}}(\operatorname{curl} \mathcal{H}-\mathcal{J}) \times \mathbf{n}=0 \quad \text { on } \Gamma_{C}, \quad \frac{\partial \mathcal{E}}{\partial t}(0)=\frac{1}{\varepsilon_{0}}\left(\operatorname{curl} \mathcal{H}_{0}-\mathcal{J}(0)\right), \quad \frac{\partial \mathcal{H}}{\partial t}(0)=-\frac{1}{\mu_{0}} \operatorname{curl} \mathcal{E}_{0} .
\end{aligned}
$$

From now on, we focus on the electric field $\mathcal{E}$ (the magnetic field $\mathcal{H}$ could be handled similarly). Provided that the charge conservation holds, and that the initial conditions are compatible with the initial values of $\rho$ and $\mathcal{J}$, one finds that the Coulomb equation $\operatorname{div}\left(\varepsilon_{0} \mathcal{E}\right)=\rho$ is a consequence of the first equation in (3). Now, if we let

$$
\mathcal{T}_{E}:=\left\{\mathcal{V} \in \mathbf{H}(\mathbf{c u r l}, \Omega): \mathcal{V} \times \mathbf{n}_{\mid \partial \Omega} \in \mathbf{L}^{2}(\partial \Omega), \mathcal{V} \times \mathbf{n}_{\mid \Gamma_{C}}=0\right\}
$$

then the first equation of (3) is equivalent to the Variational Formulation: find $\mathcal{E} \in \mathcal{T}_{E}$ s.t.

$$
\frac{\mathrm{d}^{2}}{\mathrm{~d} t^{2}}(\mathcal{E}, \mathcal{V})_{0}+c \frac{\mathrm{d}}{\mathrm{d} t}\left(\mathcal{E}_{T}, \mathcal{V}_{T}\right)_{0, \Gamma_{A}}+c^{2}(\operatorname{curl} \mathcal{E}, \operatorname{curl} \mathcal{V})_{0}=-\frac{1}{\varepsilon_{0}} \frac{\mathrm{d}}{\mathrm{d} t}(\mathcal{J}, \mathcal{V})_{0}+c \frac{\mathrm{d}}{\mathrm{d} t}\left(\mathbf{e}_{T}^{\star}, \mathcal{V}_{T}\right)_{0, \Gamma_{A}}, \quad \forall \mathcal{V} \in \mathcal{T}_{E}
$$

Above, $\mathcal{V}_{T}$ denotes the tangential part of $\mathcal{V}$ on the boundary. One can prove, following [4], that, under suitable assumptions on the data, there exists one, and only one solution to the VF (6), equal to the EM field, such that

$$
\mathcal{E} \in \mathcal{C}^{0}\left(0, T ; \mathcal{T}_{E}\right) \cap \mathcal{C}^{1}\left(0, T ; \mathbf{L}^{2}(\Omega)\right) .
$$

Then, in order to get a VF which can be discretized with the help of a continuous approximation, i.e. conforming in $\mathbf{H}^{1}(\Omega)$, let us add a $(\operatorname{div} \cdot \operatorname{div} \cdot)_{0}$ term in (6) to get an Augmented Variational Formulation. In doing so, we explicitly assume that $\rho$ belongs to $L^{2}(\Omega)$. To this end, define

$$
\begin{array}{lll}
\mathcal{X}_{E}:=\mathcal{T}_{E} \cap \mathbf{H}(\operatorname{div}, \Omega), & (\mathcal{V}, \mathcal{W})_{X}:=(\operatorname{curl} \mathcal{V}, \operatorname{curl} \mathcal{W})_{0}+(\operatorname{div} \mathcal{V}, \operatorname{div} \mathcal{W})_{0}+\left(\mathcal{V}_{T}, \mathcal{W}_{T}\right)_{0, \Gamma_{A}} ; \\
\mathcal{X}_{E}^{0}:=\mathcal{X}_{E} \cap \mathbf{H}_{0}(\operatorname{curl}, \Omega), & (\mathcal{V}, \mathcal{W})_{X^{0}}:=(\operatorname{curl} \mathcal{V}, \operatorname{curl} \mathcal{W})_{0}+(\operatorname{div} \mathcal{V}, \operatorname{div} \mathcal{W})_{0} .
\end{array}
$$

It is well-known [6] that $(\cdot, \cdot)_{X}$ (respectively $(\cdot, \cdot)_{X^{0}}$ ) defines a norm on $\mathcal{X}_{E}$ (resp. $\mathcal{X}_{E}^{0}$ ), which is equivalent to the full norm. Then, $\mathcal{E}$ is the solution to the Mixed, Augmented Variational Formulation: find $(\mathcal{E}, p) \in \mathcal{X}_{E} \times L^{2}(\Omega)$ s.t.

$$
\begin{aligned}
& \frac{\mathrm{d}^{2}}{\mathrm{~d} t^{2}}(\mathcal{E}, \mathcal{V})_{0}+c \frac{\mathrm{d}}{\mathrm{d} t}\left(\mathcal{E}_{T}, \mathcal{V}_{T}\right)_{0, \Gamma_{A}}+c^{2}(\mathcal{E}, \mathcal{V})_{X^{0}}+(p, \operatorname{div} \mathcal{V})_{0} \\
& \quad=-\frac{1}{\varepsilon_{0}} \frac{\mathrm{d}}{\mathrm{d} t}(\mathcal{J}, \mathcal{V})_{0}+\frac{c^{2}}{\varepsilon_{0}}(\rho, \operatorname{div} \mathcal{V})_{0}+c \frac{\mathrm{d}}{\mathrm{d} t}\left(\mathbf{e}_{T}^{\star}, \mathcal{V}_{T}\right)_{0, \Gamma_{A}}, \quad \forall \mathcal{V} \in \mathcal{X}_{E}, \\
& (\operatorname{div} \mathcal{E}, q)_{0}=\frac{1}{\varepsilon_{0}}(\rho, q)_{0}, \quad \forall q \in L^{2}(\Omega) .
\end{aligned}
$$

Above, the Lagrange multiplier $p$ is added, to enforce numerically the Coulomb equation, in the case when the charge conservation equation is not numerically true. For instance, when one performs simulations on the motion of charged particles, which amounts to solving the coupled Vlasov-Maxwell system of equations (see [8]). This MAVF was first introduced in [3], and it produced good results either in convex domains, or in domains with a smooth boundary. To build a converging sequence of approximation in the case of a non-convex domain with a non-smooth boundary, such has a prismatic domain with reentrant edges, one has to take into account the singular behaviour of the electric field. This is the purpose of the next sections.

To conclude this section, recall that the lifting of the tangential trace of $\mathcal{E}$ on $\Gamma_{A}$ belongs to $\mathbf{H}^{1}(\Omega)$. Thus, by removing such a lifting to the electric field, the difference $\mathcal{E}^{0}$ is in $\mathcal{X}_{E}^{0}$. For discretization purposes, it is interesting to note that $\mathcal{E}_{z}^{0}$ always belongs to $H^{1}(\Omega)$. Indeed, owing to [5,7], one can prove the 
Proposition 2.1. Let $\mathcal{V} \in \mathcal{X}_{E}^{0}$. Then $\mathcal{V}_{z}$ belongs to $H^{1}(\Omega)$, and $\partial_{z} \mathcal{V}_{x}$ and $\partial_{z} \mathcal{V}_{y}$ belong to $L^{2}(\Omega)$

In other words, in a prismatic domain of the form $\omega \times] 0, L$, only the first two components $\mathcal{E}_{x}, \mathcal{E}_{y}$ of the electric field may be singular. In addition, these two components can be singular only in the $x, y$ variables.

\section{Fourier expansion and consequences}

For $u$ in $L^{2}(\Omega)$ with $\left.\Omega=\omega \times\right] 0, L[$, one can perform Fourier expansions, such as

$$
u=\sum_{k \geqslant 0} u^{k}(x, y) \sin \left(\frac{k \pi z}{L}\right) \quad \text { or } \quad u=\sum_{k \geqslant 0} u^{k}(x, y) \cos \left(\frac{k \pi z}{L}\right), \quad \text { with }\|u\|_{0}^{2}=\frac{L}{2} \sum_{k \geqslant 0}\left\|u^{k}\right\|_{0, \omega}^{2} .
$$

In our case, we remark that, according to the boundary condition (2) on $\Gamma_{C}$, one has

$$
\mathcal{E}_{x}=\mathcal{E}_{y}=0 \quad \text { on } \Gamma_{C} \cap\{z=0\} \text { and } \Gamma_{C} \cap\{z=L\},
$$

whereas there is no condition for the third component. Thus, a natural choice is to consider

$$
\mathcal{E}_{x}=\sum_{k \geqslant 0} \mathcal{E}_{x}^{k} \sin \left(\frac{k \pi z}{L}\right), \quad \mathcal{E}_{y}=\sum_{k \geqslant 0} \mathcal{E}_{y}^{k} \sin \left(\frac{k \pi z}{L}\right), \quad \mathcal{E}_{z}=\sum_{k \geqslant 0} \mathcal{E}_{z}^{k} \cos \left(\frac{k \pi z}{L}\right) .
$$

Obviously, the same choice is made for test functions $\mathcal{V}$ of $\mathcal{X}_{E}$. This further leads to

$$
\begin{aligned}
& \operatorname{curl} \mathcal{V}=\sum_{k \geqslant 0}\left(\left[\left(\operatorname{curl}^{k} \mathcal{V}^{k}\right)_{x} \mathbf{e}_{x}+\left(\operatorname{curl}^{k} \mathcal{V}^{k}\right)_{y} \mathbf{e}_{y}\right] \cos \left(\frac{k \pi z}{L}\right)+\left(\operatorname{curl}^{k} \mathcal{V}^{k}\right)_{z} \sin \left(\frac{k \pi z}{L}\right) \mathbf{e}_{z}\right), \quad \text { with } \\
& \left(\operatorname{curl}^{k} \mathcal{V}^{k}\right)_{x}=\partial_{y} \mathcal{V}_{z}^{k}-\frac{k \pi}{L} \mathcal{V}_{y}^{k}, \quad\left(\operatorname{curl}^{k} \mathcal{V}^{k}\right)_{y}=\frac{k \pi}{L} \mathcal{V}_{x}^{k}-\partial_{x} \mathcal{V}_{z}^{k}, \quad\left(\operatorname{curl}^{k} \mathcal{V}^{k}\right)_{z}=\partial_{x} \mathcal{V}_{y}^{k}-\partial_{y} \mathcal{V}_{x}^{k} \\
& \operatorname{div} \mathcal{V}=\sum_{k \geqslant 0}\left(\operatorname{div}^{k} \mathcal{V}^{k}\right) \sin \left(\frac{k \pi z}{L}\right), \quad \text { with } \operatorname{div}^{k} \mathcal{V}^{k}=\partial_{x} \mathcal{V}_{x}^{k}+\partial_{y} \mathcal{V}_{y}^{k}-\frac{k \pi}{L} \mathcal{V}_{z}^{k}
\end{aligned}
$$

Then, one chooses

$$
p=\sum_{k \geqslant 0} p^{k} \sin \left(\frac{k \pi z}{L}\right)
$$

Now, we introduce the 2D scalar curl and div operators curl $\mathbf{v}=\partial_{x} v_{y}-\partial_{y} v_{x}$ and $\operatorname{div} \mathbf{v}=\partial_{x} v_{x}+\partial_{y} v_{y}$. Let $v$ denote the unit outward normal to $\partial \omega$, and $\tau$ the unit vector such that $(\tau, v)$ is direct. Let us split $\partial \omega=\overline{\gamma_{C}} \cap \overline{\gamma_{A}}$ and introduce the Sobolev spaces defined on $\omega$

$$
\mathbf{X}_{E}:=\left\{\mathbf{v} \in \mathbf{H}(\operatorname{curl}, \operatorname{div}, \omega): \mathbf{v} \cdot \tau_{\mid \partial \omega} \in L^{2}(\partial \omega), \mathbf{v} \cdot \tau_{\mid \gamma_{C}}=0\right\}, \quad X_{E}:=\left\{v \in H^{1}(\omega): v_{\mid \gamma_{C}}=0\right\}
$$

According to (12), (14), there holds

$$
\mathcal{V} \in \mathcal{X}_{E} \Longleftrightarrow \forall k \geqslant 0, \mathcal{V}_{x, y}^{k} \in \mathbf{X}_{E}, \mathcal{V}_{z}^{k} \in X_{E}, \quad \text { together with the ad hoc equalities of norms. }
$$

With suitable Fourier expansions of $\mathcal{J}, \rho$ and $\mathbf{e}^{\star}$, we find that the MAVF (9), (10) is equivalent to the sequence of 2D-MAVFs, for $k \geqslant 0$ : find $\left(\mathcal{E}^{k}, p^{k}\right) \in\left(\mathbf{X}_{E} \times X_{E}\right) \times L^{2}(\omega)$ s.t.

$$
\begin{aligned}
& \frac{\mathrm{d}^{2}}{\mathrm{~d} t^{2}}\left(\mathcal{E}^{k}, \mathcal{V}\right)_{0, \omega}+c \frac{\mathrm{d}}{\mathrm{d} t}\left(\mathcal{E}_{T}^{k}, \mathcal{V}_{T}\right)_{0, \gamma_{A}}+c^{2}\left(\operatorname{curl}^{k} \mathcal{E}^{k}, \operatorname{curl}^{k} \mathcal{V}\right)_{0, \omega}+c^{2}\left(\operatorname{div}^{k} \mathcal{E}^{k}, \operatorname{div}^{k} \mathcal{V}\right)_{0, \omega}+\left(p^{k}, \operatorname{div}^{k} \mathcal{V}\right)_{0, \omega} \\
& \quad=-\frac{1}{\varepsilon_{0}} \frac{\mathrm{d}}{\mathrm{d} t}\left(\mathcal{J}^{k}, \mathcal{V}\right)_{0, \omega}+\frac{c^{2}}{\varepsilon_{0}}\left(\rho^{k}, \operatorname{div}^{k} \mathcal{V}\right)_{0, \omega}+c \frac{\mathrm{d}}{\mathrm{d} t}\left(\mathbf{e}_{T}^{\star, k}, \mathcal{V}_{T}\right)_{0, \gamma_{A}}, \quad \forall \mathcal{V} \in \mathbf{X}_{E} \times X_{E} \\
& \left(\operatorname{div}^{k} \mathcal{E}^{k}, q\right)_{0, \omega}=\frac{1}{\varepsilon_{0}}\left(\rho^{k}, q\right)_{0, \omega}, \quad \forall q \in L^{2}(\omega) .
\end{aligned}
$$

NB. For $k=0$, the unknown is $\left(0,0, \mathcal{E}_{z}^{0}, 0\right)$, since only $\mathcal{E}_{z}^{0}$ is not multiplied by zero in the Fourier expansions. 


\section{The 3D Fourier Singular Complement Method}

On the one hand, owing to Proposition 2.1, one can approximate the third component $\mathcal{E}_{z}^{k}$ by the usual continuous $P_{2}$ Lagrange FE. On the other hand, the first two components $\mathcal{E}_{x, y}^{k}$ can be approximated by the 2D Singular Complement Method [2,1,10,8,11], for instance by the $P_{2}$ Lagrange FE component by component, together with a suitable Singular Complement. Finally, the Lagrange multiplier $p$ is approximated by the $P_{1}$ Lagrange FE. This yields a Taylor-Hood FE discretization, with a Singular Complement. One then proves easily the two results

Proposition 4.1. The bilinear form $(\mathcal{V}, \mathcal{W}) \mapsto\left(\operatorname{curl}^{k} \mathcal{V}, \operatorname{curl}^{k} \mathcal{W}\right)_{0, \omega}+\left(\operatorname{div}^{k} \mathcal{V}, \operatorname{div}^{k} \mathcal{W}\right)_{0, \omega}+\left(\mathcal{V}_{T}, \mathcal{W}_{T}\right)_{0, \gamma_{A}}$ is coercive on $\left(\mathbf{X}_{E} \times X_{E}\right) \times\left(\mathbf{X}_{E} \times X_{E}\right)$, for $k \geqslant 0$.

Proposition 4.2. The 2D discretized MAVF (18), (19) satisfies a uniform, discrete inf-sup condition.

NB. The discrete inf-sup condition is uniform with respect to the meshsize and to the Fourier mode.

To conclude, we introduce an approximation of the solution $(\mathcal{E}, p)$ to $(9),(10)$. Let $h$ denote the meshsize of the 2D triangulation of $\omega$, and $N$ the number of Fourier modes that are actually computed. Following [7] and the previous sections, we approximate $\mathcal{E}$ and $p$ respectively by

$$
\mathcal{E}_{\Omega}^{N, h}=\sum_{k=0}^{k=N}\left(\left(\mathcal{E}_{x}^{k, h} \mathbf{e}_{x}+\mathcal{E}_{y}^{k, h} \mathbf{e}_{y}\right) \sin \left(\frac{k \pi z}{L}\right)+\mathcal{E}_{z}^{k, h} \cos \left(\frac{k \pi z}{L}\right) \mathbf{e}_{z}\right), \quad p_{\Omega}^{N, h}=\sum_{k=0}^{k=N} p^{k, h} \sin \left(\frac{k \pi z}{L}\right),
$$

where $\left(\mathcal{E}^{k, h}, p^{k, h}\right)$ is the solution to the discretization of (18), (19). Then, combining the results of the previous sections allow us to prove that $\left(\mathcal{E}_{\Omega}^{N, h}, p_{\Omega}^{N, h}\right)_{N, h}$ converges to the solution $(\mathcal{E}, p)$ of the MAVF (9), (10) in $\mathcal{X}_{E} \times L^{2}(\Omega)$.

\section{Acknowledgements}

The first two authors acknowledge the support of the PROCORE grant. The last author acknowledges the support of Hong Kong RGC grants (project no. 403403 and CUHK4048/02P).

\section{References}

[1] F. Assous, P. Ciarlet Jr., E. Garcia, Solution of the time-dependent Maxwell equations with charges in a two-dimensional singular domain, C. R. Acad. Sci. Paris, Ser. I 330 (2000) 391-396.

[2] F. Assous, P. Ciarlet Jr., J. Segré, Numerical solution to the time-dependent Maxwell equations in two-dimensional singular domain: The Singular Complement Method, J. Comput. Phys. 161 (2000) 218-249.

[3] F. Assous, P. Degond, E. Heintzé, P.A. Raviart, J. Segré, On a finite element method for solving the three-dimensional Maxwell equations, J. Comput. Phys. 109 (1993) 222-237.

[4] F. Ben Belgacem, C. Bernardi, Spectral element discretization of the Maxwell equations, Math. Comp. 68 (1999) 1497-1520.

[5] M.Sh. Birman, M.Z. Solomyak, Maxwell operator in regions with nonsmooth boundaries, Sib. Math. J. 28 (1987) 12-24.

[6] P. Ciarlet, Jr., Augmented formulations for solving Maxwell equations, Comput. Methods Appl. Mech. Engrg., in press.

[7] P. Ciarlet, Jr., B. Jung, S. Kaddouri, S. Labrunie, J. Zou, The Fourier Singular Complement Method for the Poisson problem. Part I: prismatic domains, Numer. Math., submitted for publication.

[8] E. Garcia, PhD Thesis, Paris 6 University, France, 2002 (in French).

[9] E. Garcia, S. Labrunie, Space-time regularity of the solution to Maxwell's equations in non-convex domains, C. R. Acad. Sci. Paris, Ser. I 334 (2002) 293-298.

[10] C. Hazard, S. Lohrengel, A singular field method for Maxwell's equations: numerical aspects for 2D magnetostatics, SIAM J. Appl. Math. 40 (2002) 1021-1040.

[11] E. Jamelot, Nodal finite element methods for Maxwell's equations, C. R. Acad. Sci. Paris, Ser. I, in press. 